\title{
A phase II study of an enhanced recovery after surgery protocol in gastric cancer surgery
}

\author{
Norihiko Sugisawa $^{1,2} \cdot$ Masanori Tokunaga $^{1} \cdot$ Rie Makuuchi $^{1} \cdot$ Yuichiro Miki $^{1}$ • \\ Yutaka Tanizawa $^{1} \cdot$ Etsuro Bando $^{1} \cdot$ Taiichi Kawamura $^{1} \cdot$ Masanori Terashima $^{1}$
}

Received: 23 April 2015/Accepted: 28 July 2015/Published online: 11 August 2015

(C) The International Gastric Cancer Association and The Japanese Gastric Cancer Association 2015

\begin{abstract}
Background The enhanced recovery after surgery (ERAS) protocol integrates a number of perioperative interventions and techniques, aiming at decreasing the morbidity rate and the length of postoperative hospital stay after surgery. Although it has become a standard perioperative management for colorectal surgery, the feasibility of the ERAS protocol for gastric surgery remains unclear. Methods This single-center, prospective phase II study included patients with gastric cancer undergoing curative gastrectomy. The primary end point was the incidence of Clavien-Dindo grade II or higher postoperative complications. The secondary end points were the incidence of anastomotic leakage, the incidence of pneumonia, the proportion of patients starting oral feeding at postoperative day 2, the completion rate of the ERAS protocol, the length of postoperative hospital stay, the readmission rate within 30 days after discharge, and the mortality rate.

Results From September 2013 to September 2014, 121 eligible patients were enrolled in this study. The incidence of postoperative complications was $10.7 \%$ (90\% confidence interval, 6.47-16.54\%). Anastomotic leakage and pneumonia was observed in one and zero patients, respectively. The median length of postoperative hospital
\end{abstract}

Masanori Tokunaga

m.tokunaga@scchr.jp

1 Division of Gastric Surgery, Shizuoka Cancer Center, 1007 Shimonagakubo, Nagaizumi-cho, Sunto-gun, Shizuoka 411-8777, Japan

2 Department of Surgery, Tohoku University Graduate School of Medicine, Miyagi, Japan stay was 8 days, and the completion rate of the ERAS protocol was $85.1 \%$. The readmission rate and the mortality rate were $0 \%$.

Conclusions The ERAS protocol can be safely used in patients undergoing gastric cancer surgery. The superiority of the ERAS protocol over non-ERAS perioperative management should be clarified.

Keywords Stomach neoplasms - Gastrectomy · Perioperative care $\cdot$ Carbohydrates $\cdot$ Clinical trial

\section{Introduction}

Gastric cancer is frequently observed in East Asia, and is the third leading cause of cancer-related death worldwide [1]. A gastrectomy with lymphadenectomy is a mainstay of the treatment, and perioperative chemotherapy or chemoradiotherapy is administered in patients with advanced disease [2-5]. The morbidity rate after gastrectomy is reported to be $13.8-46.0 \%$ [6-10]. Postoperative recovery may be enhanced and the morbidity rate may be decreased with appropriate perioperative management.

The enhanced recovery after surgery (ERAS) protocol integrates a number of perioperative interventions and techniques that individually have been shown to improve outcomes [11-13]. The main components of the ERAS protocol are preoperative carbohydrate loading, early oral feeding, appropriate pain control, and early mobilization. Several prospective studies have shown the effect of the ERAS protocol to decrease the morbidity rate and the length of postoperative hospital stay after colorectal surgery [14-16]. As a result, the ERAS protocol became a standard perioperative management in patients with colorectal cancer. 
ERAS protocols contain various components and it is unclear which of these contribute strongly to enhanced recovery. Thus, omission of components may reduce the effects of the protocol. Previous prospective and retrospective studies have investigated the feasibility of ERAS protocols for gastric cancer and have reported safe indications. However, some of the potentially important components of the ERAS protocol, such as carbohydrate loading, were excluded in some studies [17-21]. Although the superiority of ERAS protocols has been favorably demonstrated in prospective randomized controlled trials in gastrectomy patients [22-25], non-ERAS management of control subjects differed significantly between studies, precluding meta-interpretations of these and the present data. In addition, no previous studies included more than 100 patients, and further studies of the feasibility of complete ERAS protocols are required to define appropriate perioperative management procedures for these patients before widespread use of the ERAS protocol.

Our standard perioperative management already included most of the ERAS components that are used in many Japanese and Korean institutes. However, liquid was allowed on the second postoperative day (POD) and oral feeding was allowed on the third POD, and preoperative carbohydrate loading, which is thought to be one of the most important components of the ERAS protocol, was not included. Therefore, we planned the present phase II study to assess the safety of earlier oral feeding (clear liquid on the first POD and a liquid diet on the second POD) and preoperative carbohydrate loading. The aim of this singlecenter, prospective study was to clarify the feasibility of the ERAS protocol for gastric cancer surgery.

\section{Materials and methods}

This study was designed as a single-center, prospective phase II trial. The Institutional Review Board of Shizuoka Cancer Center approved the study protocol, which had the following inclusion criteria: histologically confirmed adenocarcinoma of the stomach for which curative gastrectomy is expected without simultaneous resection of other organs except for the gallbladder, no involvement of the duodenum and esophagus, patient age of 20-75 years, sufficient oral intake, an Eastern Cooperative Oncology Group performance status of 0 , no contraindication for epidural anesthesia, no prior chemotherapy or radiotherapy for any malignancy, adequate organ function, and written informed consent. The study was registered with the University Hospital Medical Information Network (UMIN000011619; http://www.umin.ac.jp/ctr/).
The operative procedure and lymph node dissection were planned on the basis of the Japanese Gastric Cancer Treatment Guidelines 2010 published by the Japanese Gastric Cancer Association [26]. Patients with stage I gastric cancer were allowed to choose the surgical approach: open, laparoscopy assisted, or robot assisted. Patients with advanced disease underwent open gastrectomy. The clinical stage and residual tumor status were confirmed on the basis of the International Union Against Cancer classification system [27].

\section{ERAS protocol}

The standard perioperative management and course were explained to the patients at the outpatient clinic. The perioperative management is shown in Fig. 1. The patients were allowed to ingest a solid diet until the day before the surgery, and drank $500 \mathrm{ml}$ of a carbohydrate-rich drink (18.0 g per $100 \mathrm{ml}$ carbohydrate; Arginaid water; Nestle Health Science, Japan) after dinner. On the day of surgery, another $250 \mathrm{ml}$ was drunk up to $3 \mathrm{~h}$ before anesthesia. Neither premedication for bowel preparation nor premedication for anesthesia was administered before surgery, and the patients always went to operation room on foot. An antibiotic drug (cefazolin) was infused before skin incision every $3 \mathrm{~h}$, and infusion finished on the day of surgery. Intraoperative fluid management was basically decided by the anesthesiologist under the policy of avoiding sodium and fluid overload. The nasogastric tube was removed $3 \mathrm{~h}$ after surgery unless there were signs of bleeding. Continuous epidural analgesia with ropivacaine and opioid (fentanyl) was given for 3 days after the surgery. Additional anodyne (nonsteroidal anti-inflammatory drugs) was allowed when required by the patient. On the first POD, patients started walking and were allowed to start drinking clear fluid. On the second POD, a liquid diet was given unless patients had a high temperature $\left(38.5^{\circ} \mathrm{C}\right.$ or higher), and patients were encouraged to walk by themselves. Drainage tubes were removed on the second or third POD if the drainage fluid was clear, amylase concentrations were less than $1000 \mathrm{U} / \mathrm{l}$, and the amount of drainage discharge was less than $100 \mathrm{ml} /$ day. Removal of urinary catheters was planned for the third POD. Peripheral parenteral nutrition was continued until the fourth POD, and on the first POD, a total of $2000 \mathrm{ml}$ of $7.5 \%$ glucose fluid containing amino acids and vitamins was administered in 24 h. Subsequently, the doses of $7.5 \%$ glucose fluid were tapered off to 1500,1000 , and $500 \mathrm{ml}$ on the second, third, and fourth PODs, respectively. Routine pharmacological thromboprophylaxis was not administered unless preoperative venous ultrasonography, which was performed routinely in all patients, showed deep venous thrombosis. Patients 


\begin{tabular}{|c|c|c|c|c|c|c|c|c|c|}
\hline & \multirow[t]{2}{*}{ Day before surgery } & \multicolumn{3}{|c|}{ Day of surgery } & \multicolumn{5}{|c|}{ Day after surgery } \\
\hline & & Before operation & During operation & After operation & Day 1 & Day 2 & Day 3 & Day 4 & Day 5-10 \\
\hline Ambulation & Free & Free & & Bed rest & Early mobilization & $\rightarrow$ & $\rightarrow$ & Free & $\rightarrow$ \\
\hline Carbohydrate & $90 \mathrm{~g} / 500 \mathrm{ml}$ & $45 \mathrm{~g} / 250 \mathrm{ml}$ & & & & & & & \\
\hline Oral intake & Normal diet & $\begin{array}{c}\text { Clear fluids up to } 3 \\
\text { hour before } \\
\text { anesthesia }\end{array}$ & & Nothing & Clear fluids & Liquid diet & Soft meal & $\rightarrow$ & $\rightarrow$ \\
\hline Transfusion & & & Fluid management & Crystalloids & \begin{tabular}{c|} 
Peripheral \\
parenteral nutrition
\end{tabular} & $\rightarrow$ & $\rightarrow$ & Finish & \\
\hline Bowel preparation & None & & & & & & & & \\
\hline Preanesthetic medication & None & & & & & & & & \\
\hline Antimicrobial prophylaxis & & & $\begin{array}{c}\text { Before skin incision } \\
\text { and every } 3 \mathrm{~h}\end{array}$ & Once & & & & & \\
\hline Nasogastric tube & & & Insert & Remove & & & & & \\
\hline Drainage tube & & & Insert & $\rightarrow$ & $\rightarrow$ & Ren & nove & & \\
\hline Urinary catheter & & & Insert & $\rightarrow$ & $\rightarrow$ & $\rightarrow$ & Remove & & \\
\hline Epidural analgesia & & & Start & $\rightarrow$ & $\rightarrow$ & $\rightarrow$ & Finish & & \\
\hline
\end{tabular}

Fig. 1 Enhanced recovery after surgery protocol in gastric cancer surgery

were discharged between the seventh and the tenth POD if the attending surgeon judged the amount of food intake was enough and patients did not have any complications requiring in-hospital treatment.

\section{End points}

The primary end point in this study was the incidence of postoperative complications. Patients who developed Clavien-Dindo classification [28] grade II or higher morbidities by discharge were regarded as having postoperative complications. The secondary end points were the incidence of anastomotic leakage, the incidence of pneumonia, the proportion of patients starting oral feeding on the second POD, the completion rate of the ERAS protocol, the length of postoperative hospital stay, the readmission rate within 30 days after discharge, and the mortality rate.

\section{Study design and statistical methods}

In this phase II trial, 107 patients were required, providing a $90 \%$ power under the hypothesis of a primary end point with an expected value of $10 \%$ and a threshold of $20 \%$, with two-sided testing at the $10 \%$ significance level. The expected value was decided according to the postoperative outcome of 181 patients matching the eligibility criteria of the present study at Shizuoka Cancer Center in 2011; the incidence of postoperative complications among these patients was $12.7 \%$. All statistical analyses were conducted with $\mathrm{R}$ version 3.1.0.

\section{Results}

At the outpatient clinic, 128 patients agreed to be enrolled in this phase II study from September 2013 to September 2014. However, 5 of 128 patients were found to be ineligible for the ERAS protocol before admission (two because of a contraindication for epidural anesthesia, one because of a mental disorder, one because of prior chemotherapy, and one because an adenocarcinoma was not found in by a biopsy specimen). In addition, the carbohydrate-rich drink could not be prepared for two patients; therefore, these patients were considered to be ineligible at the time of admission. The remaining 121 patients were managed with the ERAS protocol and were included in this analysis.

Table 1 summarizes the patient characteristics. The median age was 64 years. The clinical stage was IA or IB in $86 \%$ of patients (104 of 121).

The surgical findings are shown in Table 2. Distal gastrectomy was the most frequently performed procedure (62.8\%), followed by pylorus-preserving gastrectomy $(25.6 \%)$ and total gastrectomy $(8.3 \%)$. D1+ lymph node dissection was performed in 88 patients $(72.7 \%)$, and D2 dissection was performed in 33 patients $(27.3 \%)$. The laparoscopic approach was the most preferred approach (37.2\%), followed by the robot-assisted approach (32.2\%) and the open approach $(30.6 \%)$.

Early surgical outcomes and details of postoperative complications are shown in Tables 3 and 4. Thirteen postoperative complications developed in 121 patients (13 of 121 , $10.7 \%$; $90 \%$ confidence interval, 6.47-16.54\%). ClavienDindo grade IIIa complications were observed in three patients (anastomotic leakage with abscess, pancreas fistula with abscess, and intra-abdominal bleeding). Anastomotic leakage was observed after distal gastrecotmy with Billroth I reconstruction. Clavien-Dindo grade IIIb or higher complications were not observed in any of the patients. The median length of postoperative hospital stay was 8 days. Four patients were discharged from the hospital on the sixth POD, which is earlier than the planned discharge day, because of the patients' requirements. None of the patients were readmitted to the hospital within 30 days after discharge. 
Table 1 Patient characteristics

\begin{tabular}{|c|c|}
\hline Number of patients & 121 \\
\hline \multicolumn{2}{|l|}{ Age (years) } \\
\hline Median & 64 \\
\hline Range & $24-74$ \\
\hline \multicolumn{2}{|l|}{ Sex (cases) } \\
\hline Male & 78 \\
\hline Female & 43 \\
\hline \multicolumn{2}{|l|}{ BMI $\left(\mathrm{kg} / \mathrm{m}^{2}\right)$} \\
\hline Median & 22.5 \\
\hline Range & $16.5-29.6$ \\
\hline \multicolumn{2}{|c|}{ ECOG performance status (cases) } \\
\hline 0 & 121 \\
\hline $1 / 2$ & 0 \\
\hline \multicolumn{2}{|l|}{ Clinical stage (cases) } \\
\hline IA & 91 \\
\hline IB & 13 \\
\hline IIA & 7 \\
\hline IIB & 4 \\
\hline IIIA & 3 \\
\hline IIIB & 2 \\
\hline IIIC & 1 \\
\hline IV & 0 \\
\hline
\end{tabular}

BMI body mass index, ECOG Eastern Cooperative Oncology Group

Post hoc subgroup analyses were performed to identify factors that were associated with the development of postoperative complications. However, none of the covariates investigated, including male $(9 / 78,11.5 \%)$ versus female $(4 / 43,9.3 \%)$, early gastric cancer $(12 / 104,11.5 \%)$ versus advanced gastric cancer $(1 / 17,5.9 \%)$, total gastrectomy ( $0 /$ $10,0 \%$ ) versus other procedures $(13 / 111,11.7 \%)$, D2 dissection $(2 / 33,6.1 \%)$ versus $\mathrm{D} 1+$ dissection $(11 / 88$, $12.5 \%)$, and open surgery $(4 / 37,10.8 \%$ ) versus laparoscopy-assisted or robot-assisted surgery $(9 / 84,10.7 \%)$, affected the postoperative complications significantly.

Table 5 shows the completion rate of the ERAS protocol. Preoperative carbohydrate loading was accomplished in 119 patients $(98.3 \%)$, and two patients could not drink $500 \mathrm{ml}$ of the carbohydrate-rich drink on the day before the surgery. Early oral feeding was accomplished in 113 patients $(93.4 \%)$. Six patients started oral feeding on the third POD or later because of a postoperative complication (four patients) or the attending surgeon's judgment (two patients). Of the 121 patients, nine patients $(7.4 \%)$ could not be discharged by the tenth POD because of postoperative complications, and 112 patients $(92.6 \%)$ were discharged from the hospital on the tenth POD or earlier. The completion rate of whole ERAS protocol was $85.1 \%$ (103 of 121 patients).
Table 2 Surgical treatment

\begin{tabular}{lr}
\hline Type of gastrectomy $(n)$ & 76 \\
Distal gastrectomy & 31 \\
Pylorus-preserving gastrectomy & 4 \\
Proximal gastrectomy & 10 \\
Total gastrectomy & 88 \\
Lymph node dissection (cases) & 33 \\
D1+ & \\
D2 & 113 \\
Combined resection (cases) & 7 \\
None & 1 \\
Gallbladder & 0 \\
Colon & \\
Spleen & 37 \\
Surgical approach (cases) & 45 \\
Open & 39 \\
Laparoscopy assisted & \\
Robot assisted & 121 \\
Residual tumor (cases) & 0 \\
0 & \\
1/2 &
\end{tabular}

Table 3 Early surgical outcomes

\begin{tabular}{ll}
\hline Blood loss (ml) & 30 \\
Median & $0-728$ \\
Range & \\
Operation time (min) & 290 \\
Median & $109-486$ \\
Range & 13 \\
Postoperative complications ${ }^{\text {a }}(n)$ & 0 \\
Postoperative hospital deaths (cases) & \\
Length of postoperative hospital stay (days) & 8 \\
Median & $6-30$ \\
Range & 0 \\
Hospital readmissions $(n)$ & \\
\hline
\end{tabular}

${ }^{a}$ Clavien-Dindo classification grade II or higher

\section{Discussion}

This phase II study showed that the ERAS protocol, including early oral feeding in gastric cancer surgery, is feasible in terms of safety. The incidence of postoperative complications was $10.7 \%$ (13 of 121 patients), and the upper limit of the $90 \%$ confidence interval was $16.54 \%$ and was lower than the threshold of $20 \%$; thus, the null hypothesis was rejected.

Carbohydrate loading is an important component of the ERAS protocol and is thought to be effective to avoid degradation of protein and enhance postoperative recovery 
Table 4 Postoperative complications (Clavien-Dindo classification grade II or higher)

\begin{tabular}{lllllll}
\hline Complications & Total number & Grade II & Grade IIIa & Grade IIIb & Grade IV & Grade V \\
\hline Anastomotic leakage & 1 & 0 & 1 & 0 & 0 & 0 \\
Pneumonia & 0 & 0 & 0 & 0 & 0 & 0 \\
Pancreatic fistula & 2 & 1 & 1 & 0 & 0 & 0 \\
Intra-abdominal abscess & 3 & 1 & 2 & 0 & 0 & 0 \\
Distal gastric emptying & 2 & 2 & 0 & 0 & 0 & 0 \\
Bowel obstruction & 1 & 1 & 0 & 0 & 0 & 0 \\
Bleeding & 2 & 1 & 1 & 0 & 0 & 0 \\
Cerebral infarction & 1 & 1 & 0 & 0 & 0 & 0 \\
Liver dysfunction & 3 & 3 & 0 & 0 & 0 & 0 \\
Diverticulitis & 1 & 1 & 0 & 0 & 0 & 0 \\
\hline
\end{tabular}

Table 5 Compliance with the enhanced recovery after surgery $(E R A S)$ protocol

\begin{tabular}{lll}
\hline ERAS protocol components & $\begin{array}{l}\text { Number of } \\
\text { patients }\end{array}$ & $\begin{array}{l}\text { Percentage } \\
\text { of patients }\end{array}$ \\
\hline Preoperative carbohydrate loading & 119 & 98.3 \\
Removal of epidural anesthesia & 121 & 100 \\
Removal of nasogastric tube & 119 & 98.3 \\
Early mobilization & 118 & 97.5 \\
Early oral feeding & 113 & 93.4 \\
Removal of drainage tube & 107 & 88.4 \\
Removal of urinary catheter & 121 & 100 \\
Discharge as scheduled & 108 & 89.3 \\
All components & 103 & 85.1 \\
\hline
\end{tabular}

$[19,29]$; however, it also has a potential drawback. Carbohydrate loading enhances intestinal movement [30], resulting in an adverse effect on the anastomotic site, particularly after upper gastrointestinal tract surgery. In addition, early oral feeding after surgery may also increase the incidence of anastomotic leakage and aspiration pneumonia. In this study, therefore, the incidence of anastomotic leakage and that of pneumonia were set as secondary end points.

The incidence of anastomotic leakage was $0.8 \%$, and was as low as that reported in previous studies $(0.8-1.9 \%)$ based on conventional perioperative management, and was lower than that in eligible subjects of our historical cohort $(1.7 \%)[6,8,31]$. Therefore, we thought that carbohydrate loading and early oral feeding did not harmfully affect the anastomotic site. Yamada et al. [18, 32] evaluated the value of ERAS protocols in a Japanese high-volume center and reported low incidence of anastomotic leakage (1.1-1.5\%) as in the present series.

Another potential drawback of the ERAS protocol is aspiration pneumonia, but it was not observed in any of the patients in this study. Previous reports on ERAS protocols for colorectal cancer showed that respiratory complications developed in $7.8 \%$ of patients (184 of 2352 patients) [33]. Similarly, Feng et al. [22] reported an incidence of pneumonia in $8.5 \%$ of patients who received early oral feeding on the first POD. However, others reported no pneumonia after initiation of oral feeding on the second POD after gastrectomy [18, 25, 32, 34, 35]. Therefore, we concluded that the incidence of aspiration pneumonia does not increase with the use of ERAS protocols after gastric cancer surgery.

In the present study, the severity as well as the incidence of the complications was acceptable. Of 13 patients with postoperative complications, most complications were Clavien-Dindo grade II, and were managed with medications. Although three patients $(2.5 \%)$ developed Clavien-Dindo grade IIIa complications and required intervention under local anesthesia, Clavien-Dindo grade IIIb (surgical intervention under general anesthesia), Clavien-Dindo grade IV (organ failure requiring intensive care), and Clavien-Dindo grade V (death) complications were not observed in any of the patients. Therefore, the ERAS protocol was safely introduced in terms of the severity of the complications.

In our study, $85.1 \%$ of the patients completed the ERAS protocol, and this is as high as the reported completion rate after colorectal cancer or gastric cancer surgery, which was reported as $71.5-94.5 \%$ in previous studies $[18,33,36]$. Therefore, we think that the current form of the ERAS protocol is also suitable for patients undergoing gastrectomy.

The median length of postoperative hospital stay was 8 days in this study, and was compatible with previous reports from East Asia [18, 25, 32, 35]. However, it is longer than in reports from the West [17], presumably owing to differences in insurance systems among the countries. Because the readmission rate within 30 days after discharge was zero, the discharge date of 7-10 days set in the ERAS protocol is appropriate after gastrectomy. In addition, four patients were discharged on the sixth POD, earlier than expected. Therefore, we may be able to further shorten the duration of postoperative hospital stay in the future. 
In comparisons of the present study with our historical cohort (120 patients) of eligible subjects who underwent gastrectomy in 2012, the incidences of complications $(10.7 \%$ in this study, and $11.7 \%$ in the historical cohort), the mortality rates ( $0 \%$ in each group), and the readmission rates ( $0 \%$ in each group) did not differ. However, the median duration of postoperative hospital stay was significantly shorter in the present study ( 8 days) than in the historical cohort ( 10 days; $p<0.001)$. Hence, the ERAS protocol may shorten the postoperative hospital stay without increasing the incidence of postoperative complications. Accordingly, postoperative recovery was enhanced by the present ERAS protocol. However, further prospective comparative studies comparing ERAS and non-ERAS perioperative management in sufficient numbers of patients are required to confirm these assertions.

In the present study, patient eligibility was limited to younger patients with good performance status (0); thus, the feasibility of the ERAS protocol for fragile patients remains unclear. Moreover, early oral feeding did not increase the risk of aspiration pneumonia in this study but may cause aspiration pneumonia in elderly patients with poor performance status. Secondly, the number of patients with advanced gastric cancer or those undergoing total gastrectomy was limited in this study; therefore, the feasibility of the ERAS protocol in patients with advanced gastric cancer or that in those undergoing total gastrectomy remains unclear. Yamada et al. [32] also reported the feasibility of ERAS protocols in relatively young patients with early-stage gastric cancer undergoing elective gastric surgery. Therefore, further studies of the feasibility of ERAS protocols for fragile patients or patients with advancedstage gastric cancer are required.

In conclusion, the ERAS protocol can be safely used in patients undergoing gastric cancer surgery. The superiority of the ERAS protocol over non-ERAS perioperative management should be clarified.

Acknowledgments This research was partially supported by the National Cancer Center Research and Development Fund (26-A-4) and Practical Research for Innovative Cancer Control (\#201438039A) from the Japan Agency for Medical Research and Development.

\section{Compliance with ethical standards}

Conflict of Interest The authors declare that they have no conflict of interest.

Research involving Human and Animal Rights All procedures followed were in accordance with the ethical standards of the responsible committee on human experimentation (institutional and national) and with the Helsinki Declaration of 1964 and later versions. Informed consent or substitute for it was obtained from all patients for their being included in the study.

\section{References}

1. Ferlay J, Soerjomataram I, Dikshit R, Eser S, Mathers C, Rebelo $\mathrm{M}$, et al. Cancer incidence and mortality worldwide: sources, methods and major patterns in GLOBOCAN 2012. Int J Cancer. 2015;136:E359-86.

2. Noh SH, Park SR, Yang HK, Chung HC, Chung IJ, Kim SW, et al. Adjuvant capecitabine plus oxaliplatin for gastric cancer after D2 gastrectomy (CLASSIC): 5-year follow-up of an openlabel, randomised phase 3 trial. Lancet Oncol. 2014;15:1389-96.

3. Smalley SR, Benedetti JK, Haller DG, Hundahl SA, Estes NC, Ajani JA, et al. Updated analysis of SWOG-directed intergroup study 0116: a phase III trial of adjuvant radiochemotherapy versus observation after curative gastric cancer resection. J Clin Oncol. 2012;30:2327-33.

4. Sakuramoto S, Sasako M, Yamaguchi T, Kinoshita T, Fujii M, Nashimoto A, et al. Adjuvant chemotherapy for gastric cancer with S-1, an oral fluoropyrimidine. N Engl J Med. 2007;357:1810-20.

5. Cunningham D, Allum WH, Stenning SP, Thompson JN, Van de Velde CJ, Nicolson M, et al. Perioperative chemotherapy versus surgery alone for resectable gastroesophageal cancer. $\mathrm{N}$ Engl $\mathrm{J}$ Med. 2006;355:11-20.

6. Kim HH, Han SU, Kim MC, Hyung WJ, Kim W, Lee HJ, et al. Long-term results of laparoscopic gastrectomy for gastric cancer: a large-scale case-control and case-matched Korean multicenter study. J Clin Oncol. 2014;32:627-33.

7. Sasako M, Sano T, Yamamoto S, Sairenji M, Arai K, Kinoshita $\mathrm{T}$, et al. Left thoracoabdominal approach versus abdominaltranshiatal approach for gastric cancer of the cardia or subcardia: a randomised controlled trial. Lancet Oncol. 2006;7:644-51.

8. Sano T, Sasako M, Yamamoto S, Nashimoto A, Kurita A, Hiratsuka M, et al. Gastric cancer surgery: morbidity and mortality results from a prospective randomized controlled trial comparing D2 and extended para-aortic lymphadenectomy-Japan Clinical Oncology Group study 9501. J Clin Oncol. 2004;22:2767-73.

9. Cuschieri A, Fayers P, Fielding J, Craven J, Bancewicz J, Joypaul $\mathrm{V}$, et al. Postoperative morbidity and mortality after D1 and D2 resections for gastric cancer: preliminary results of the MRC randomised controlled surgical trial. Lancet. 1996;347:995-9.

10. Bonenkamp JJ, Songun I, Hermans J, Sasako M, Welvaart K, Plukker JT, et al. Randomised comparison of morbidity after D1 and D2 dissection for gastric cancer in 996 Dutch patients. Lancet. 1995;345:745-8.

11. Basse L, Jakobsen DH, Billesbolle P, Werner M, Kehlet HA. A clinical pathway to accelerate recovery after colonic resection. Ann Surg. 2000;232:51-7.

12. Gustafsson UO, Scott MJ, Schwenk W, Demartines N, Roulin D, Francis N, et al. Guidelines for perioperative care in elective colonic surgery: Enhanced Recovery After Surgery (ERAS®) Society recommendations. Clin Nutr. 2012;31:783-800.

13. Nygren J, Thacker J, Carli F, Fearon KC, Norderval S, Lobo DN, et al. Guidelines for perioperative care in elective rectal/pelvic surgery: Enhanced Recovery After Surgery (ERAS $\left.{ }^{\circledR}\right)$ Society recommendations. Clin Nutr. 2012;31:801-16.

14. Spanjersberg WR, Reurings J, Keus F, van Laarhoven CJ. Fast track surgery versus conventional recovery strategies for colorectal surgery. Cochrane Database Syst Rev. 2011;(2):CD007635.

15. Varadhan KK, Neal KR, Dejong CH, Fearon KC, Ljungqvist O, Lobo DN. The enhanced recovery after surgery (ERAS) pathway for patients undergoing major elective open colorectal surgery: a meta-analysis of randomized controlled trials. Clin Nutr. 2010;29:434-40. 
16. Muller S, Zalunardo MP, Hubner M, Clavien PA, Demartines N. A fast-track program reduces complications and length of hospital stay after open colonic surgery. Gastroenterology. 2009;136:842-7.

17. Grantcharov TP, Kehlet H. Laparoscopic gastric surgery in an enhanced recovery programme. Br J Surg. 2010;97:1547-51.

18. Yamada T, Hayashi $\mathrm{T}$, Cho $\mathrm{H}$, Yoshikawa $\mathrm{T}$, Taniguchi $\mathrm{H}$, Fukushima R, et al. Usefulness of enhanced recovery after surgery protocol as compared with conventional perioperative care in gastric surgery. Gastric Cancer. 2012;15:34-41.

19. Awad S, Varadhan KK, Ljungqvist O, Lobo DN. A meta-analysis of randomised controlled trials on preoperative oral carbohydrate treatment in elective surgery. Clin Nutr. 2013;32:34-44.

20. Fearon KC, Jenkins JT, Carli F, Lassen K. Patient optimization for gastrointestinal cancer surgery. Br J Surg. 2013;100:15-27.

21. Mortensen K, Nilsson M, Slim K, Schafer M, Mariette C, Braga $\mathrm{M}$, et al. Consensus guidelines for enhanced recovery after gastrectomy: Enhanced Recovery After Surgery (ERAS $\left.{ }^{\circledR}\right)$ Society recommendations. Br J Surg. 2014;101:1209-29.

22. Feng F, Ji G, Li JP, Li XH, Shi H, Zhao ZW, et al. Fast-track surgery could improve postoperative recovery in radical total gastrectomy patients. World J Gastroenterol. 2013;19:3642-8.

23. Chen Hu J, Xin Jiang L, Cai L, Tao Zheng H, Yuan Hu S, Bing Chen $\mathrm{H}$, et al. Preliminary experience of fast-track surgery combined with laparoscopy-assisted radical distal gastrectomy for gastric cancer. J Gastrointest Surg. 2012;16:1830-939.

24. Liu XX, Jiang ZW, Wang ZM, Li JS. Multimodal optimization of surgical care shows beneficial outcome in gastrectomy surgery. J Parenter Enteral Nutr. 2010;34:313-21.

25. Wang D, Kong Y, Zhong B, Zhou X, Zhou Y. Fast-track surgery improves postoperative recovery in patients with gastric cancer: a randomized comparison with conventional postoperative care. J Gastrointest Surg. 2010;14:620-7.

26. Japanese Gastric Cancer Association. Japanese gastric cancer treatment guidelines 2010 (ver. 3). Gastric Cancer. 2011;14:113-23.
27. Edge SB, Byrd D, Compton CC, Fritz AG, Greene FL, Trotti A, editors. AJCC cancer staging manual. 7th ed. New York: Springer; 2010.

28. Dindo D, Demartines N, Clavien PA. Classification of surgical complications: a new proposal with evaluation in a cohort of 6336 patients and results of a survey. Ann Surg. 2004;240:205-13.

29. Svanfeldt M, Thorell A, Hausel J, Soop M, Rooyackers O, Nygren J, et al. Randomized clinical trial of the effect of preoperative oral carbohydrate treatment on postoperative wholebody protein and glucose kinetics. Br J Surg. 2007;94:1342-50.

30. Noblett SE, Watson DS, Huong H, Davison B, Hainsworth PJ, Horgan AF. Pre-operative oral carbohydrate loading in colorectal surgery: a randomized controlled trial. Colorectal Dis. 2006;8:563-9.

31. Katai H, Sasako M, Fukuda H, Nakamura K, Hiki N, Saka M, et al. Safety and feasibility of laparoscopy-assisted distal gastrectomy with suprapancreatic nodal dissection for clinical stage I gastric cancer: a multicenter phase II trial (JCOG 0703). Gastric Cancer. 2010;13:238-44.

32. Yamada T, Hayashi T, Aoyama T, Shirai J, Fujikawa H, Cho H, et al. Feasibility of enhanced recovery after surgery in gastric surgery: a retrospective study. BMC Surg. 2014;14:41.

33. ERAS Compliance Group. The impact of enhanced recovery protocol compliance on elective colorectal cancer resection: results from an international registry. Ann Surg. 2015;261:1153-9.

34. Suehiro T, Matsumata T, Shikada Y, Sugimachi K. Accelerated rehabilitation with early postoperative oral feeding following gastrectomy. Hepatogastroenterology. 2004;51:1852-5.

35. Hur H, Kim SG, Shim JH, Song KY, Kim W, Park CH, et al. Effect of early oral feeding after gastric cancer surgery: a result of randomized clinical trial. Surgery. 2011;149:561-8.

36. Haverkamp MP, de Roos MA, Ong KH. The ERAS protocol reduces the length of stay after laparoscopic colectomies. Surg Endosc. 2012;26:361-7. 